\title{
Integrated Multiplex Sensing Bandage for in situ Monitoring of Early Infected Wounds
}

Ziqi Liu ${ }^{l, *}$, Junqing Liü, ${ }^{2, *}$, Tiancheng Sun ${ }^{l}$, Deke Zeng ${ }^{l}$, Chengduan Yang ${ }^{l}$, Hao Wang ${ }^{l}$, Cheng Yang ${ }^{l}$, Jun Guo ${ }^{2}$, Qianni Wu ${ }^{3}$, Hui-Jiuan Chen ${ }^{l, ~}{ }^{*}, X i X_{i} e^{l, *}$

${ }^{1}$ School of Electronics and Information Technology, State Key Laboratory of Optoelectronic Materials and Technologies, Guangdong Province Key Laboratory of Display Material and Technology, Sun Yat-Sen University, Guangzhou 510006, China.

${ }^{2}$ Department of Cardiology, The First Affiliated Hospital of Jinan University, Guangzhou 510630, China.

${ }^{3}$ State Key Laboratory of Ophthalmology, Zhongshan Ophthalmic Center, Sun Yat-sen University, Guangzhou, 510060 China.

* Corre-sponding authors: chenhuix5@mail.sysu.edu.cn, xiexi27@mail.sysu.edu.cn

These authors contributed equally to this work. 
This file includes 10 Supplementary Figures.

Figure S1. The custom mobile application for data integrated display.

Figure S2. Fluorescence images showed the adhesion of S. aureus on the junction of the patches and the substrate.

Figure S3. The HE staining images of normal wound and infect-ed wounds 1-3.

Figure S4. The $\mathrm{CD}^{+} 5^{+}$staining images of normal wound and infected wounds 1-3 and their statistical analysis of inflammatory cell densities.

Figure S5. Electrochemical curves of deposition PEDOT:PSS and chitosan/Prussian blue mediator layer.

Figure S6. Schematic diagram of the $\mathrm{Na}^{+}, \mathrm{K}^{+}, \mathrm{Ca}^{2+}$, and $\mathrm{pH}$ signal conditioning circuits.

Figure S7. Schematic diagram of the uric acid, temperature signal conditioning circuits and the interface of the MSA.

Figure S8. Schematic diagram of the STM32F103RCT6 micro-controller with minimum system circuits.

Figure S9. Schematic diagram of USB to serial port circuit based on CH340E and Bluetooth circuit based on RF-BM4044B4 module.

Figure S10. Illustration and photograph of the power supply module. 


\section{(a)}

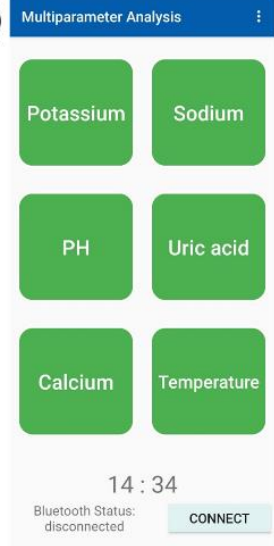

(b)

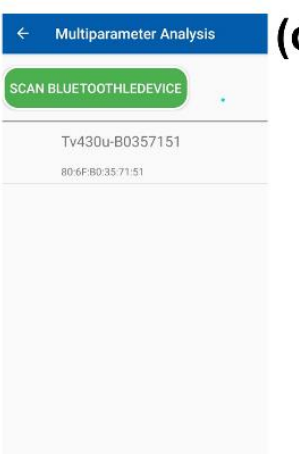

(c)

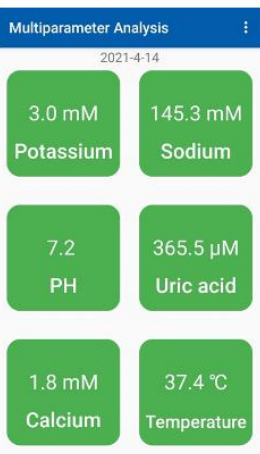

$15: 23$

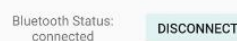

(d)

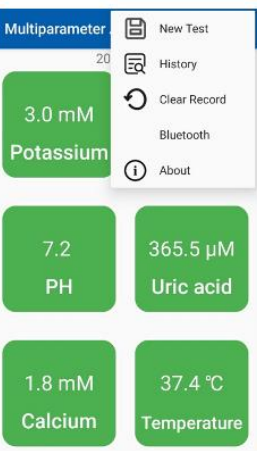

$15: 23$

Bluetooth Status: DISCONNECT
conocected (e)

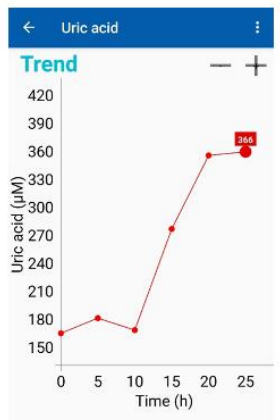

$15: 20: 52 \quad 365.50 \quad \mu \mathrm{M}$

Figure S1. The custom mobile application for data integrated display. (a) The main interface before Bluetooth connection. (b) Bluetooth connection interface. (c) The main interface of real-time data display after Bluetooth connection. (d) Additional functions including starting a new test, viewing historical data, clearing records, and Bluetooth configuration were implemented in the main interface.

(e) The sub-interface for displaying trends of individual indicators.

(a)

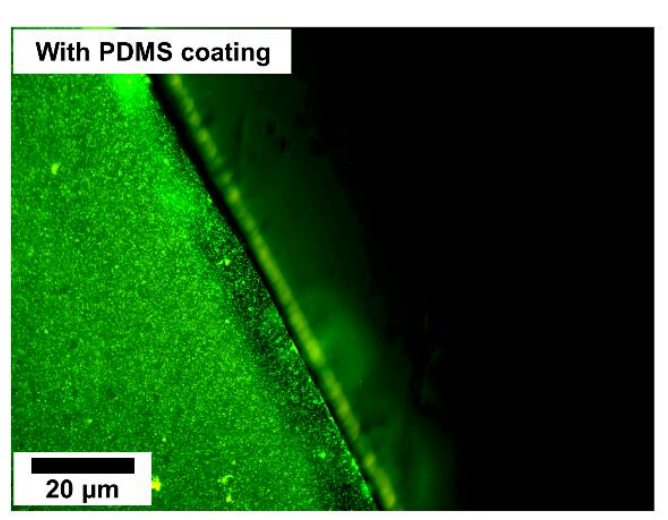

(b)

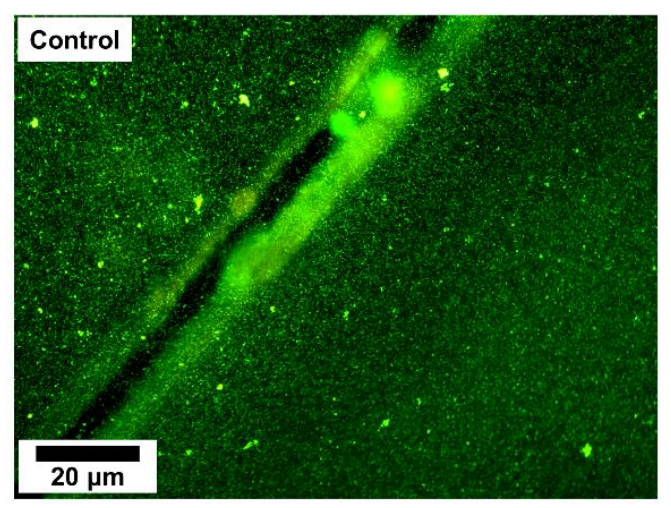

Figure S2. Fluorescence images showed the adhesion of S. aureus (stained with green fluorescence) on the junction of the patches and the substrate. (a) Fluorescence image of the junction of the PDMS coat-modified patch (left side) and the substrate (right side). (b) Fluorescence image of the junction of the uncoated patch (left side) and the substrate (right side). 

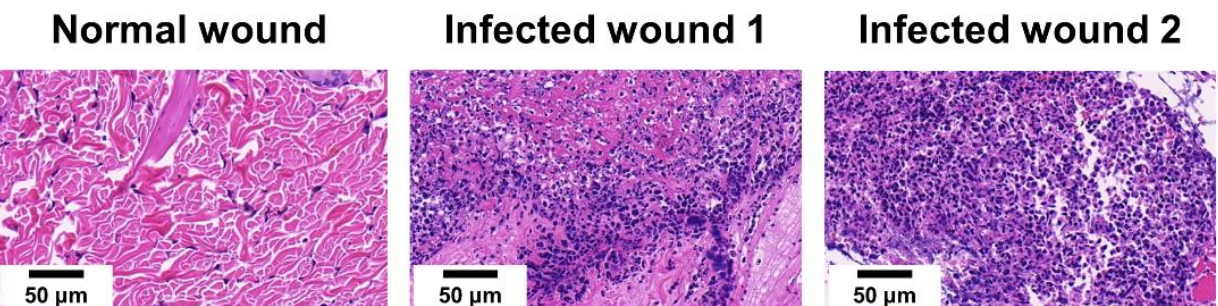

\section{Infected wound 3}
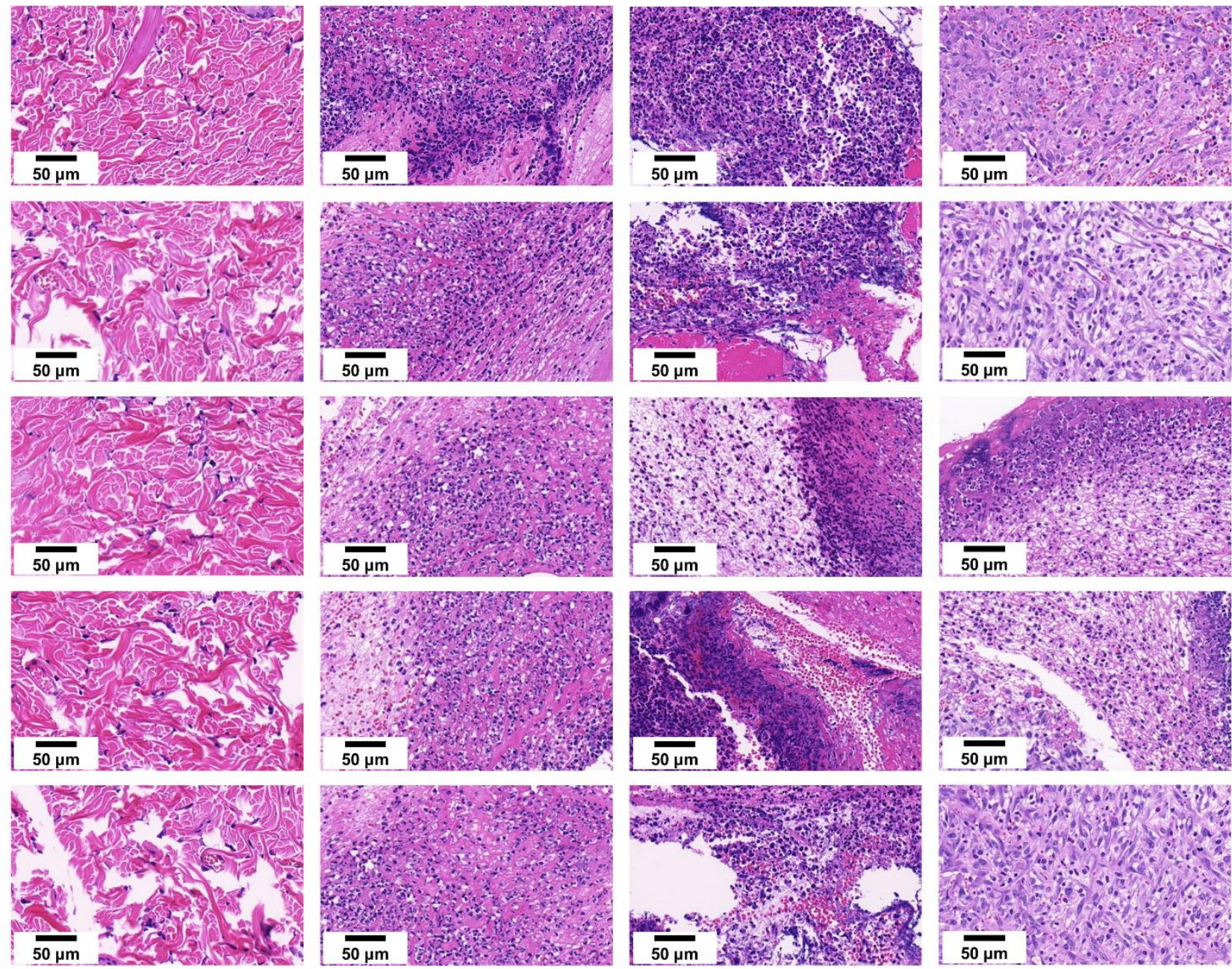

Figure S3. The HE staining images of normal wound and infected wounds 1-3. 
(a)
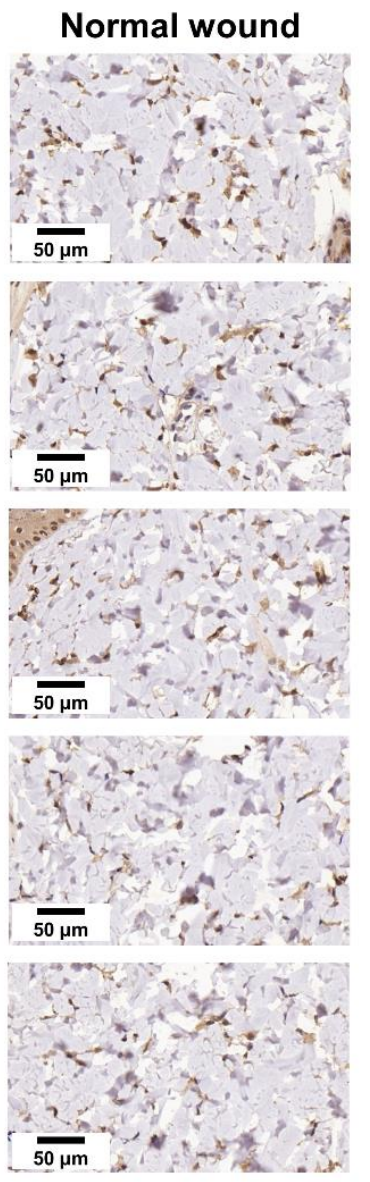

Infected wound 1
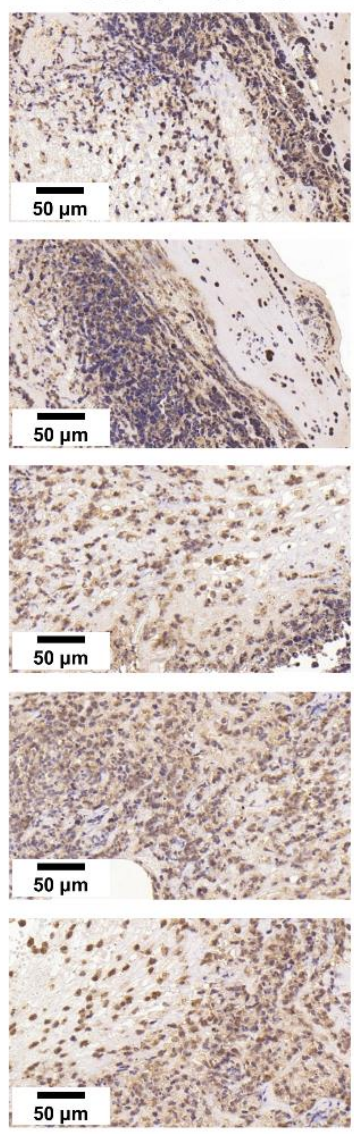

Infected wound 2
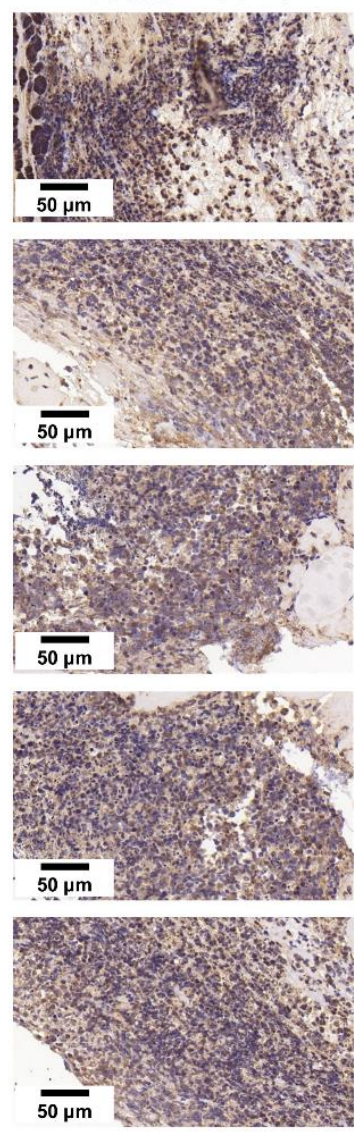

Infected wound 3
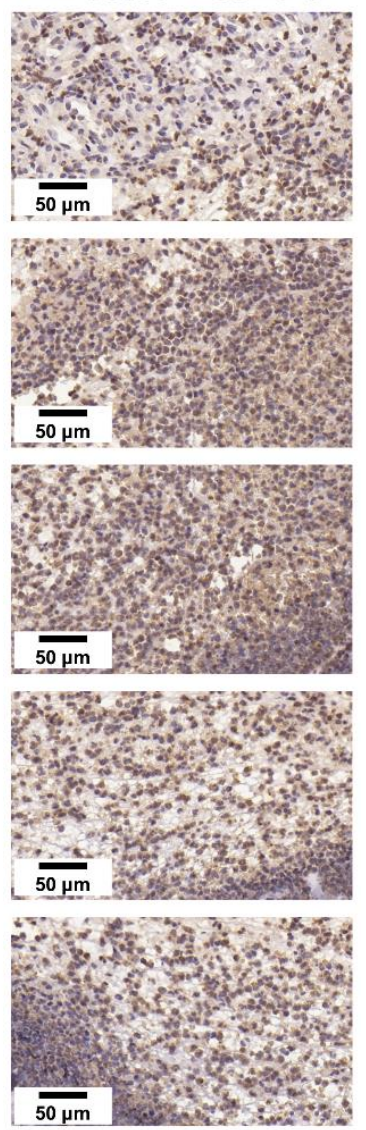

(b)

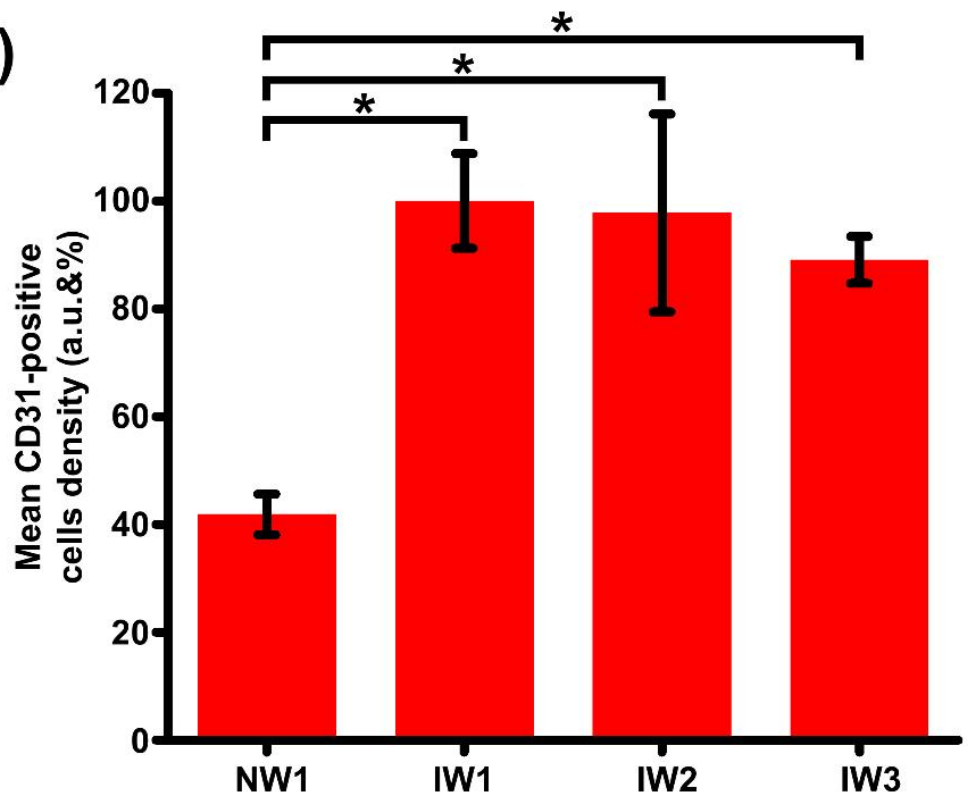

Figure S4. The CD45 ${ }^{+}$staining images of (a) normal wound (NW) and infected wounds (IW) 1-3 and (b) their statistical analysis of inflammatory cell densities. 

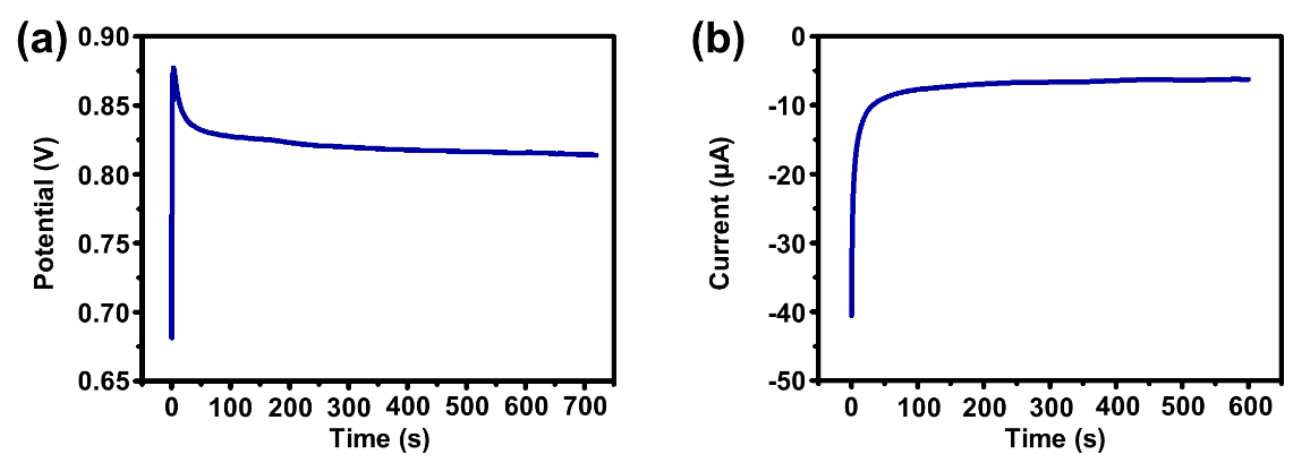

Figure S5. Electrochemical curves of deposition (a) PEDOT:PSS and (b) chitosan/Prussian blue mediator layer.

(a)

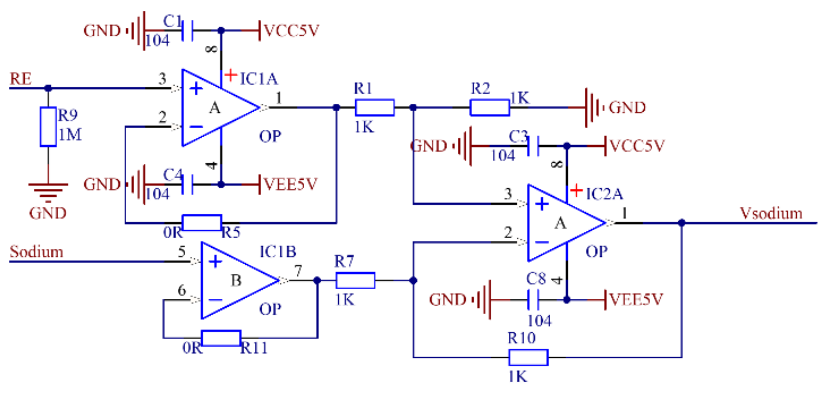

(b)

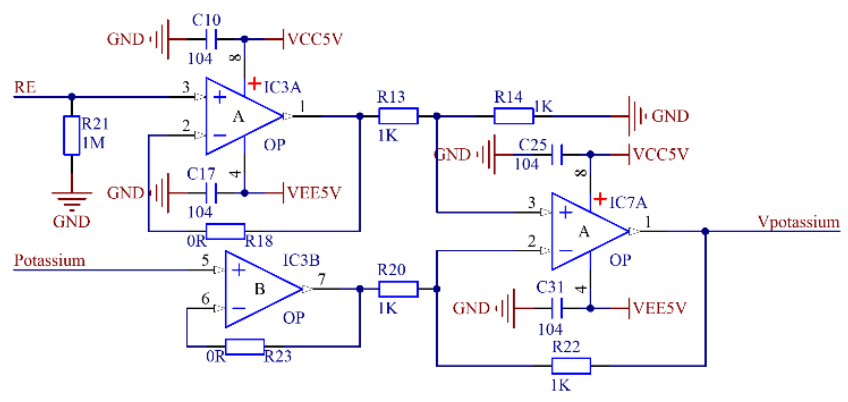

(c)

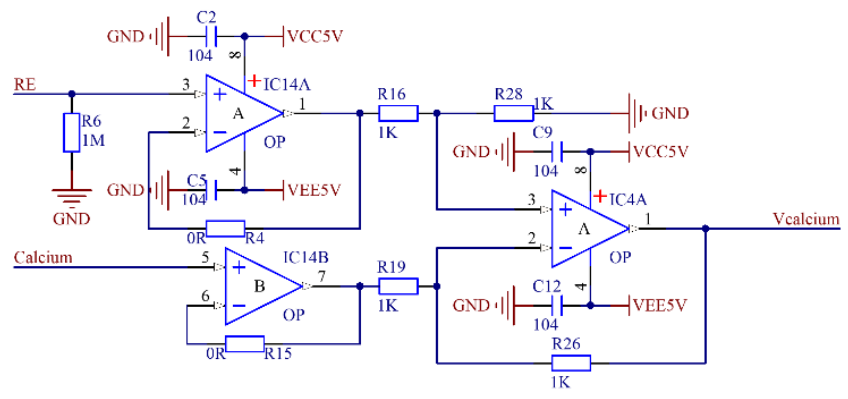

(d)

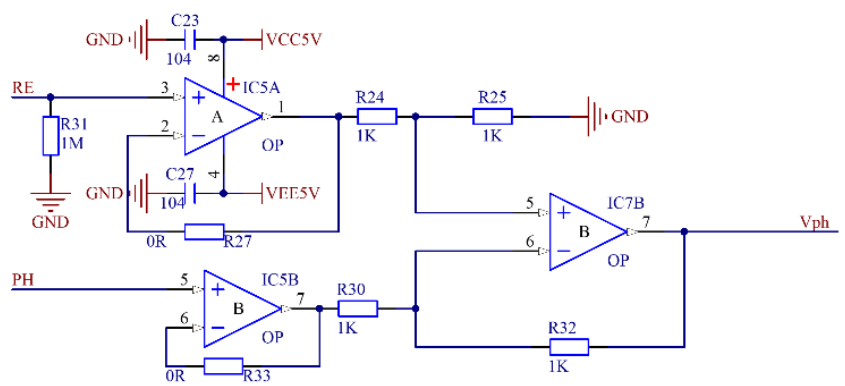

Figure S6. Schematic diagram of the (a) $\mathrm{Na}^{+}$, (b) $\mathrm{K}^{+}$, (c) $\mathrm{Ca}^{2+}$, and (d) $\mathrm{pH}$ signal conditioning circuits. 

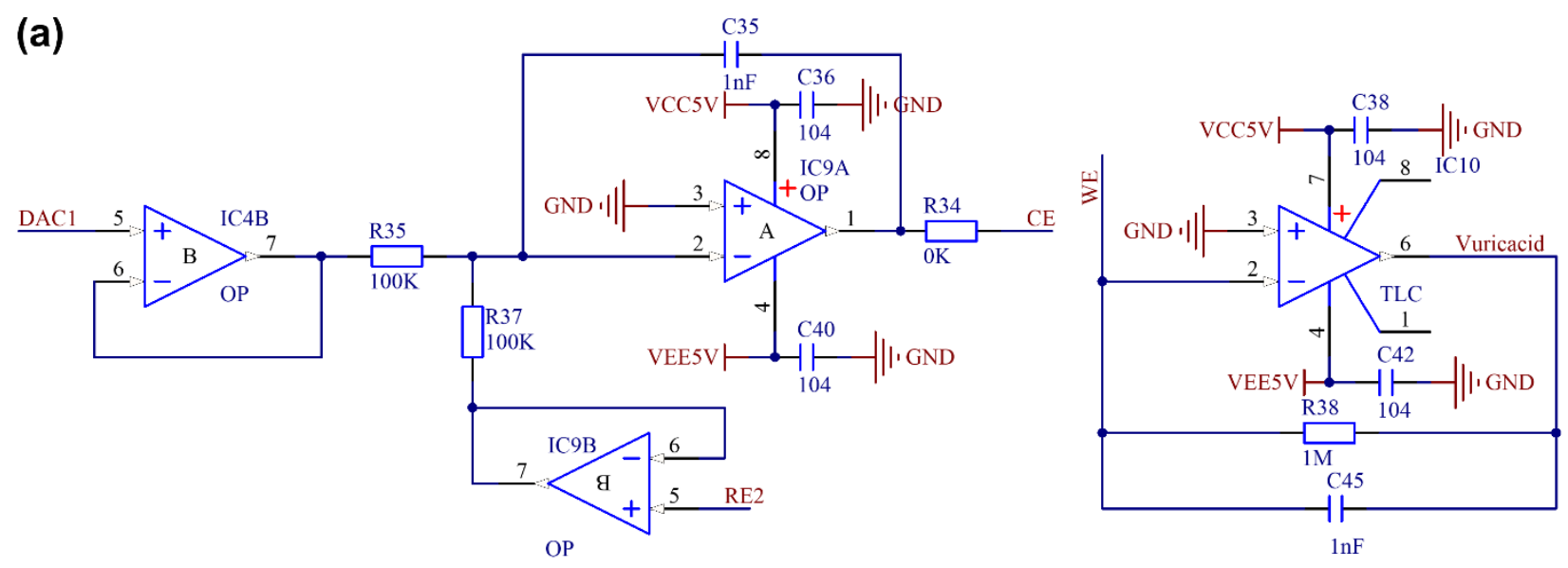

(b)

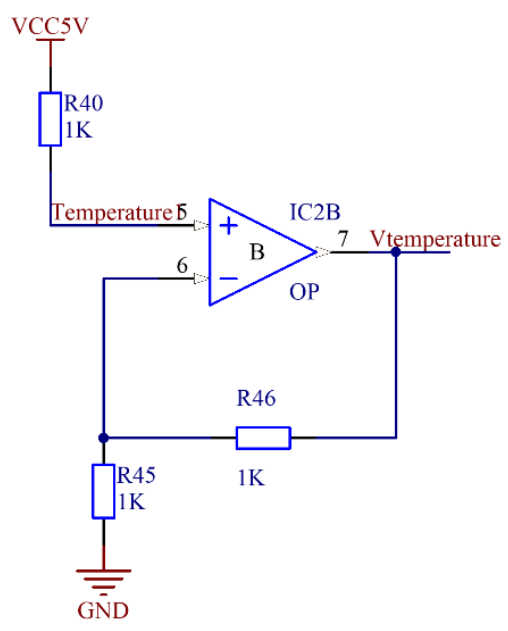

(c)

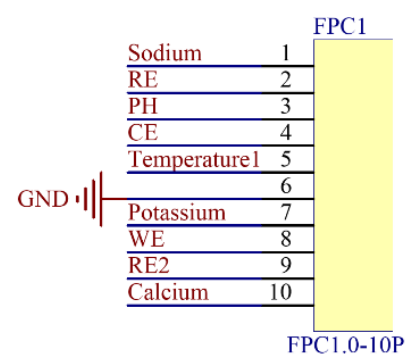

Figure S7. Schematic diagram of the (a) uric acid, (b) temperature signal conditioning circuits and (c) the interface of the MSA. 


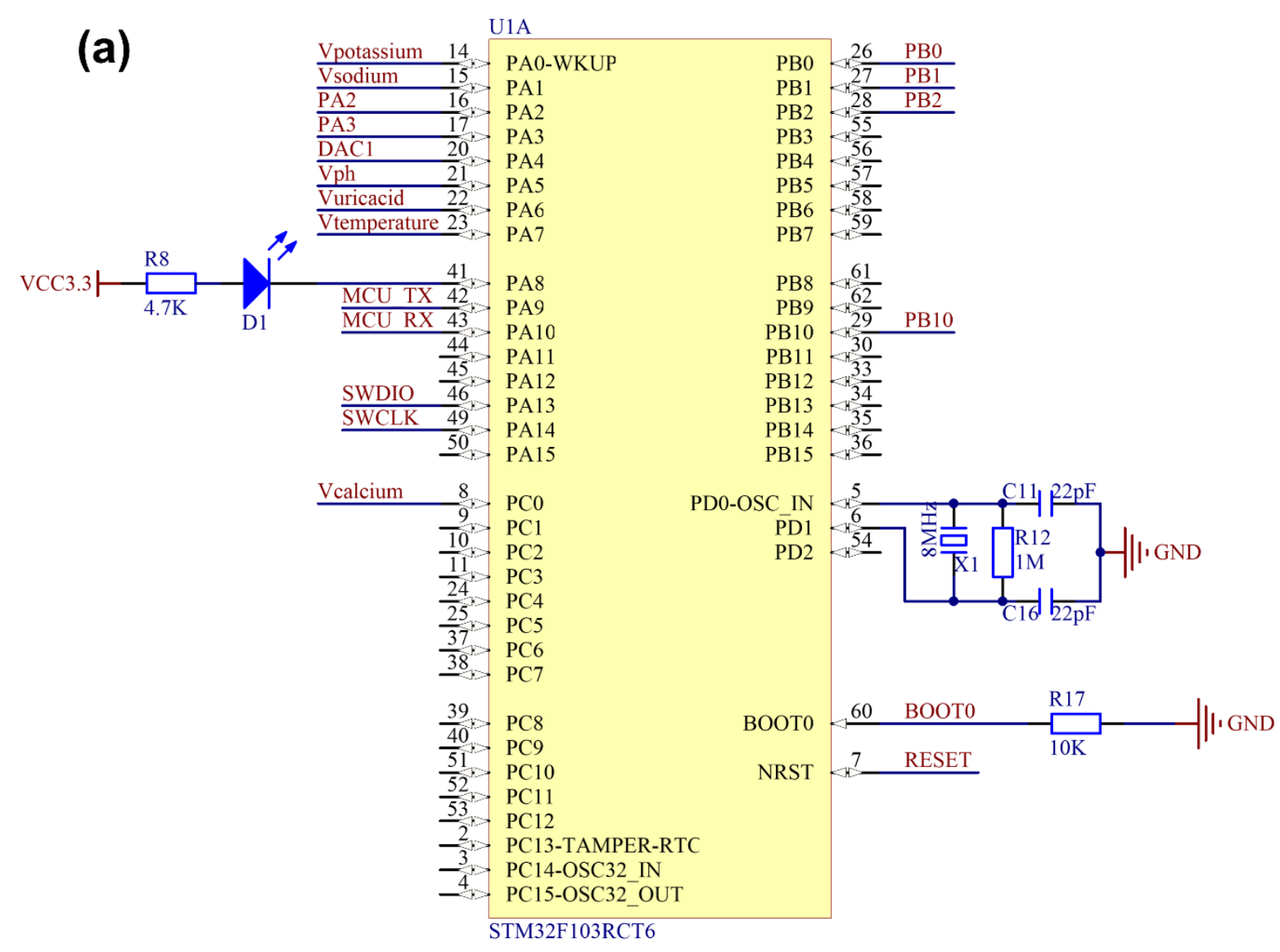

(b)

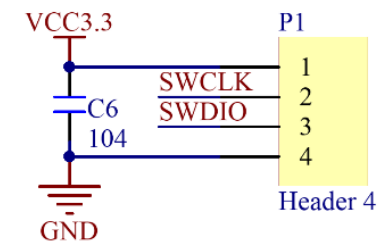

(c)

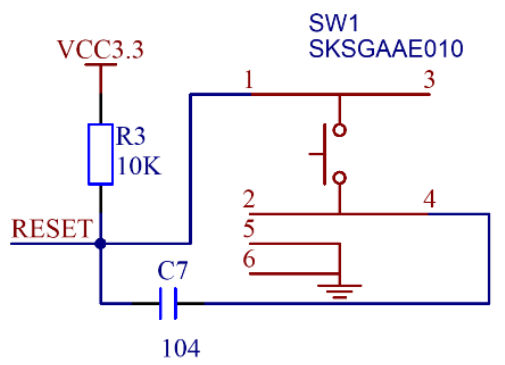

(d)

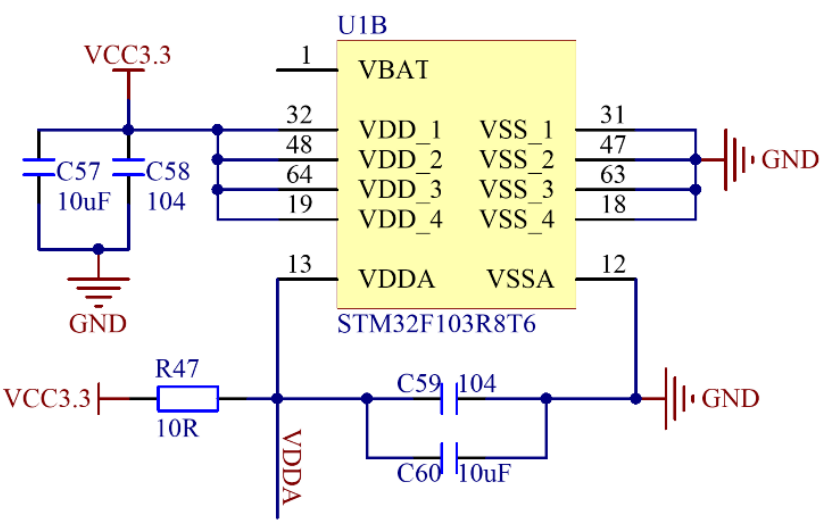

Figure S8. Schematic diagram of the STM32F103RCT6 microcontroller with minimum system circuits. (a) An external crystal oscillator circuit with an LED indicator, (b) ST-Link circuit, (c) reset circuit, (d) $3.3 \mathrm{~V}$ power supply circuit with filter. 
(a)
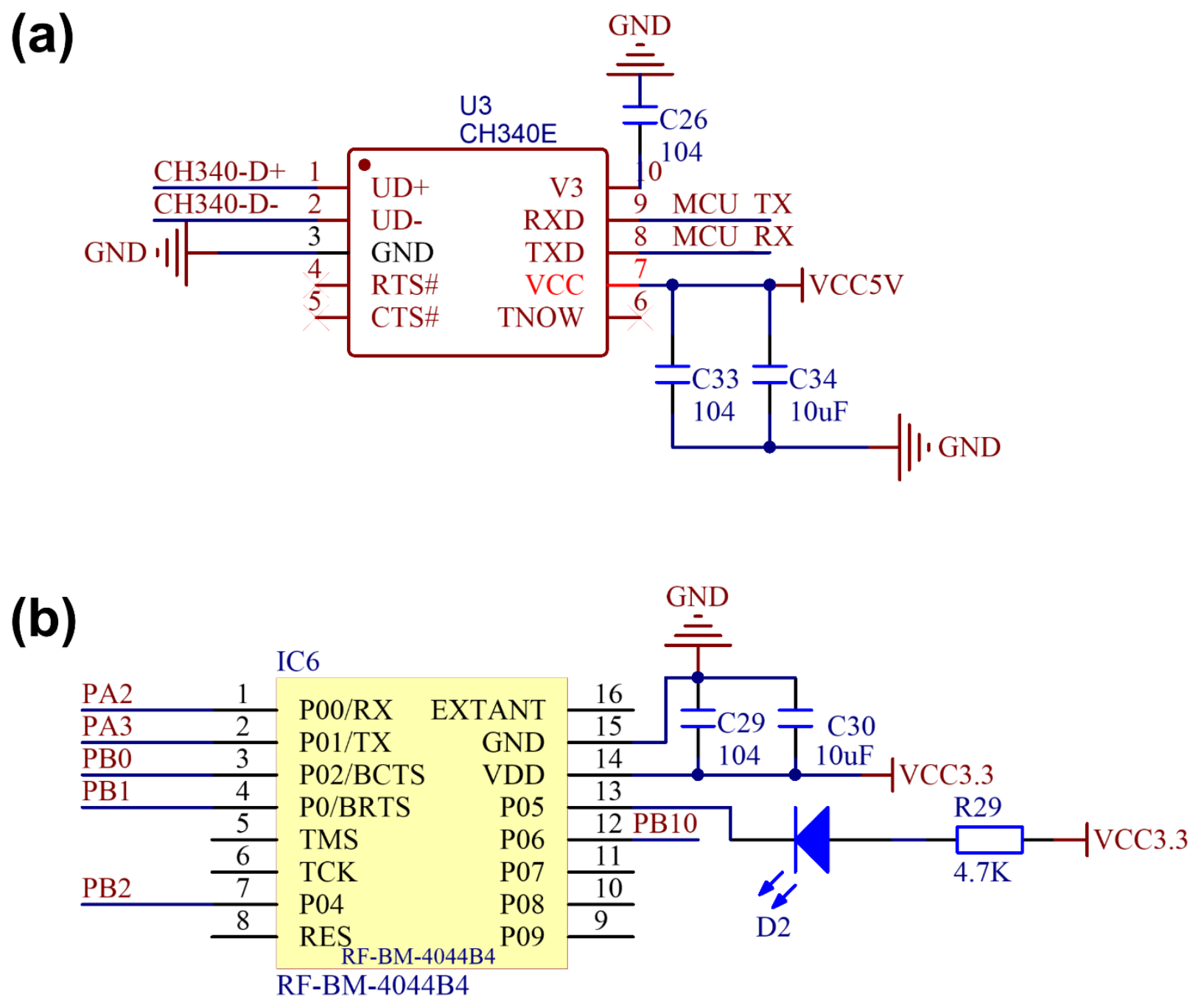

Figure S9. Schematic diagram of (a) USB to serial port circuit based on CH340E and (b) Bluetooth circuit based on RF-BM-4044B4 module. 
(a)

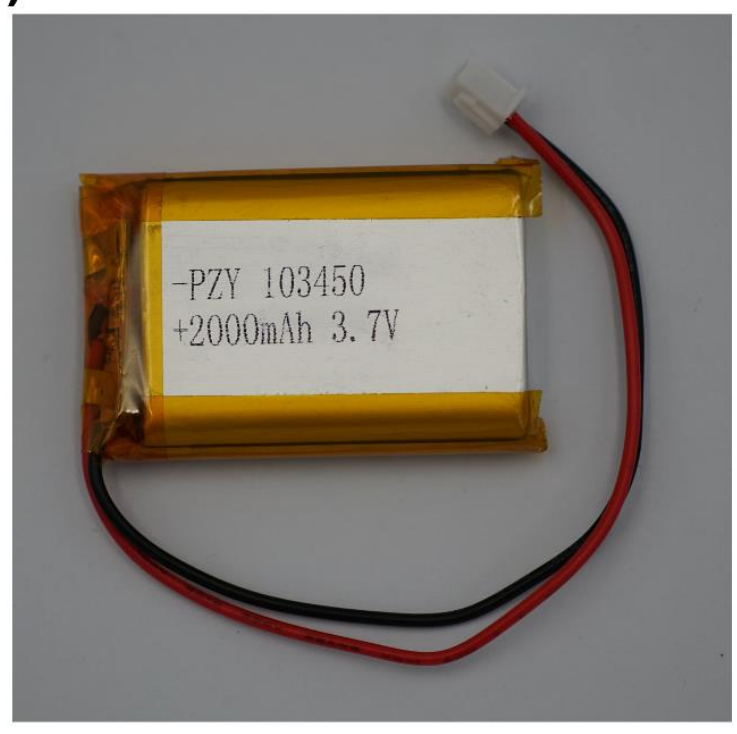

(b)

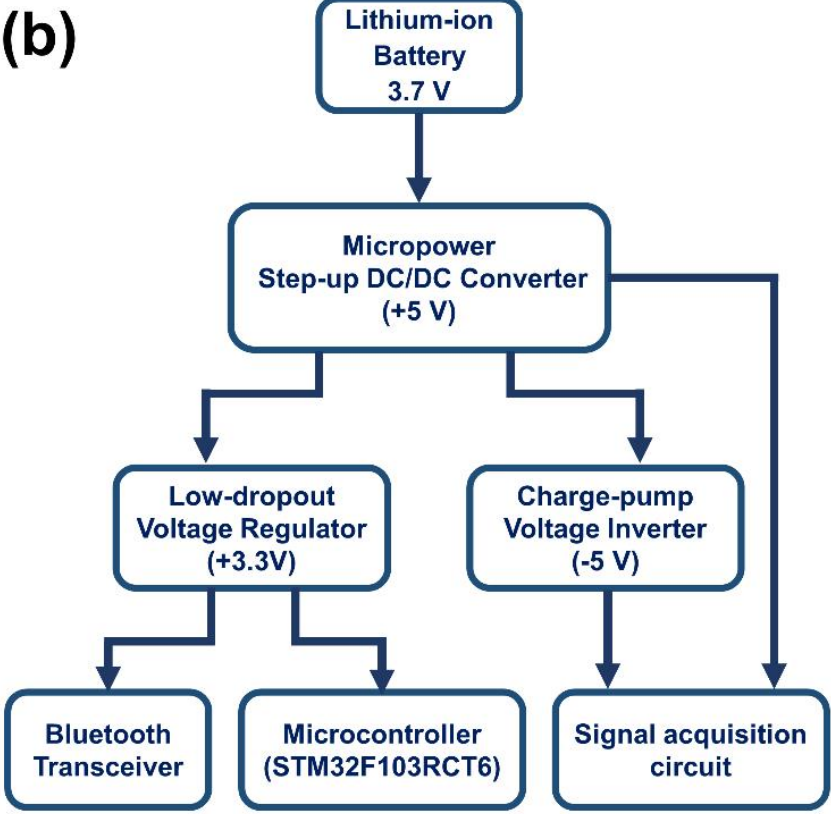

(c)
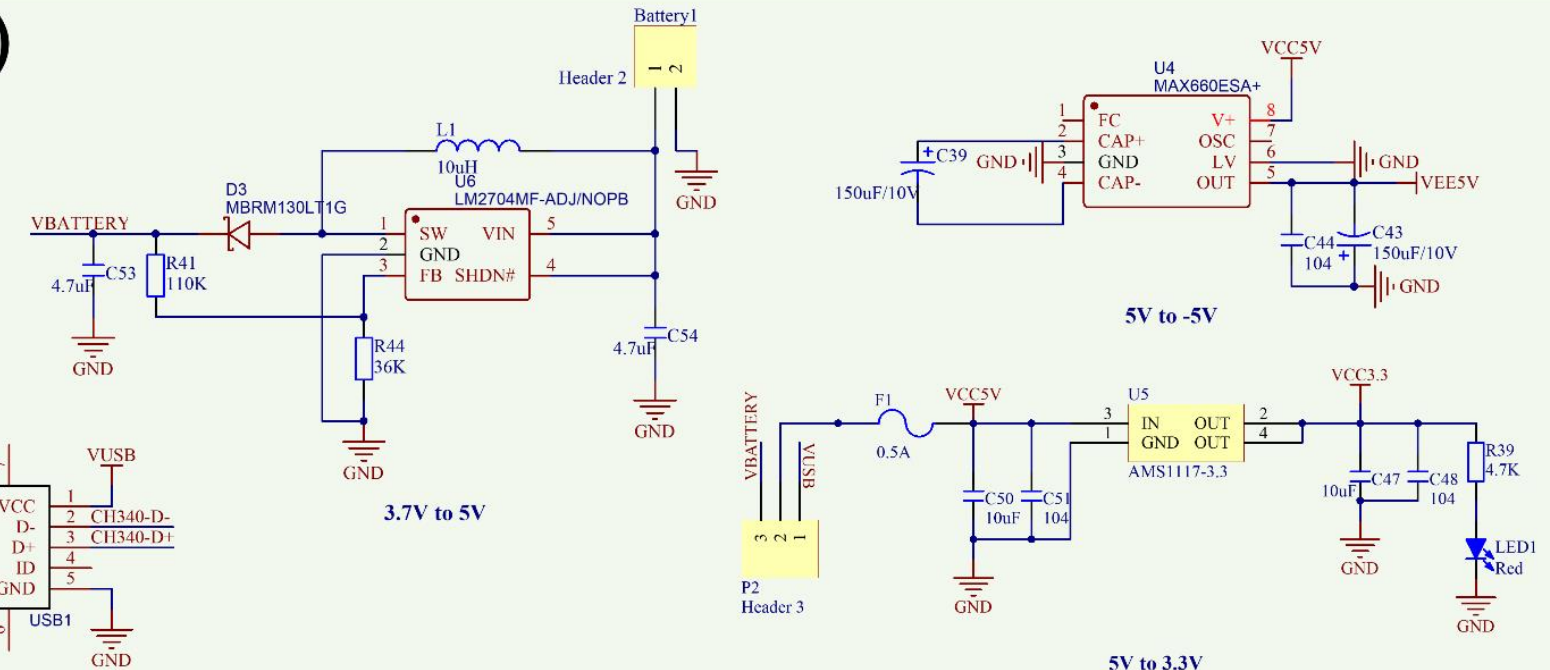

$5 \mathrm{~V}$ to $3.3 \mathrm{~V}$

Figure S10. Illustration and photograph of the power supply module. (a) Photograph of a small lithium battery used in the current work. (b) The power supply schematic diagram and (c) circuit schematic of the FIMPSB system. 